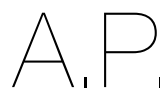

ARTIGO PROFSSIONAL

1 Faculdade de Ciências da Nutrição e Alimentação da Universidade do Porto, Rua Dr. Roberto Frias, 4200-465 Porto, Portugal

2 Unidade Local de Saúde da Guarda, E.P.E., Av. Rainha D. Amélia, S/N, 6300-858 Guarda,

Portugal

3 Unidade Local de Saúde do Alto Minho,

Estrada de Santa Luzia,

4904-858 Viana do

Castelo, Portugal

${ }^{4}$ College of Pharmacy and Nutrition of University of Saskatchewan \&

Saskatchewan Health Authority,

2A20.01 Health Sciences, 107 Wiggins Road Saskatoon SK, S7N 5E5, Canada

The New York Academy of Sciences,

7 World Trade Center 250 Greenwich Street, 40th floor New York,

NY 10007-2157, United

States of America

${ }^{6}$ Nutrition Translational Research,

69, Marmora Road

SE22ORY, London, United

Kingdom

7 Centro Hospitalar

Universitário do Algarve,

Unidade de Portimão,

Estrada do Poço Seco,

8500-338 Portimão,

Portugal

${ }^{8}$ Hospital do Divino Espírito Santo de Ponta

Delgada, E.P.E.,

Av. D. Manuel I,

9500-370 Ponta Delgada,

Portugal

${ }^{9}$ Centro Hospitalar do Funchal,

Av. Luís de Camões, n. ${ }^{\circ} 6180$ 9000-177 Funchal,

Portugal

*Endereço para correspondência:

Teresa F Amaral

Faculdade de Ciências da

Nutrição e Alimentação,

Rua Dr. Roberto Frias,

4200-465 Porto

tamaral@fcna.up.pt

Histórico do artigo:

Recebido a 10 de fevereiro de 2020 Aceite a 31 de março de 2020

\title{
DESENVOLVIMENTO DE UMA VERSÃO PORTUGUESA DO NUTRITIONAL RISK SCREENING - NRS 2002
}

\author{
DEVELOPMENT OF A PORTUGUESE VERSION OF \\ NUTRITIONAL RISK SCREENING - NRS 2002
}

Teresa F Amaral' ${ }^{1}$; Luís Matos²; Maria da Graça Ferro ${ }^{3}$; Luiza Kent-Smith; Filomena Gomes ${ }^{5}$; Susana Couto Irving ${ }^{6}$; Ana Paula Alves ${ }^{7}$; Rita Brotas Carvalho:; Maria Amélia Teixeira'; Nuno Borges'

\section{RESUMO}

O Nutritional Risk Screening (NRS 2002) é um instrumento que foi desenvolvido pela Danish Society for Parenteral and Enteral Nutrition. Trata-se de um sistema válido que permite detetar a presença do risco de desnutrição ou de desnutrição em indivíduos hospitalizados e que é aplicado pelos profissionais de saúde. Procedeu-se ao desenvolvimento de uma versão para a língua Portuguesa do NRS 2002 com equivalência linguística e cultural ao original, recorrendo à metodologia proposta pela Organização Mundial da Saúde "Processo de tradução e de adaptação de instrumentos". Realizou-se uma tradução avançada e a retrotradução, através das seguintes etapas: tradução (1. ${ }^{a}$ etapa), retrotradução efetuada por um ou mais especialistas ( $2{ }^{a}{ }^{a}$ etapa), pré-teste ( 3 . $^{\mathrm{a}}$ etapa) e preparação da versão final (4. ${ }^{a}$ etapa). Este artigo tem como objetivo divulgar este processo e também a versão Portuguesa do NRS 2002.

\section{PALAVRAS-CHAVE}

Desnutrição, NRS 2002, Rastreio, Risco nutricional, Versão portuguesa

\section{ABSTRACT}

The Nutritional Risk Screening (NRS 2002) is a tool that was developed by the Danish Society for Parenteral and Enteral Nutrition. It is a valid system that allows the detection of the risk of undernutrition or of undernutrition in hospitalized individuals and is applied by health professionals. A Portuguese language version of NRS 2002 was developed with linguistic and cultural equivalence to the original using the WHO proposed methodology "Process of translation and adaptation of instruments". Advanced translation and

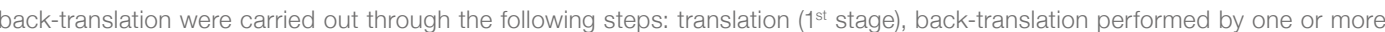
specialists ( $2^{\text {nd }}$ stage), pre test ( $3^{\text {rd }}$ stage) and preparation of the final version ( $4^{\text {th }}$ stage). This article aims to describe this process and also the Portuguese version of NRS 2002

KEYWORDS

Undernutrition, NRS 2002, Screening, Nutritional risk, Portuguese version

\section{INTRODUÇÃO}

O Nutritional Risk Screening (NRS 2002) é um instrumento que foi desenvolvido pela Danish Society for Parenteral and Enteral Nutrition. Trata-se de um sistema que permite detetar a presença do risco de desnutrição ou de desnutrição em indivíduos hospitalizados e que é aplicado pelos profissionais de saúde (1). Baseia-se no conceito de que o suporte nutricional é indicado, e poderá trazer benefícios clínicos, em doentes que estão gravemente doentes, com necessidades nutricionais aumentadas, ou com ingestão alimentar diminuída, ou que estão gravemente desnutridos, ou que têm certos graus de gravidade da doença em combinação com certos graus de desnutrição (1).

Este instrumento é constituído por dois grupos de perguntas, com pontuação definida, em que o primeiro se refere à deterioração do estado nutricional e o segundo à gravidade da doença. O primeiro grupo inclui a perda de peso não intencional recente, a redução da ingestão alimentar recente não intencional e o valor do Índice de Massa Corporal (IMC). O segundo grupo compreende uma escala de gravidade da doença: ausente, ligeira, moderada ou grave. Se a idade for igual ou superior a setenta anos, adiciona-se mais um ponto à pontuação final. O doente estará em risco nutricional quando a pontuação final for igual ou superior a três. O instrumento contém ainda quatro questões de pré rastreio para serem usadas em serviços de internamento e/ou departamentos com poucos doentes em risco (1).

Este instrumento apresenta várias vantagens quando comparado com outros disponíveis, nomeadamente o facto de o seu desenvolvimento ter sido baseado em evidência científica de elevada qualidade (2). Outras vantagens são o facto de ser aplicável a adultos de todas as idades, ter demonstrado elevada sensibilidade e especificidade em diferentes contextos, abranger todas as condições patológicas, e ser de fácil e rápida aplicação 
(2). Foi já documentada a fiabilidade, validade concorrente e preditiva do NRS 2002 (1-4), inclusive em Portugal (5, 6). Recentemente, um estudo experimental realizado em doentes médicos hospitalizados com o risco nutricional identificado pelo NRS 2002, demonstrou que o uso de suporte nutricional individualizado em comparação com a alimentação hospitalar padrão durante o internamento, melhorou os resultados clínicos e diminuiu a probabilidade de óbito no período de 30 dias (4). Estes dados vêm reforçar o conceito do rastreio sistemático no momento da admissão hospitalar, seguido por uma avaliação nutricional e pela introdução de suporte nutricional individualizado nos doentes em risco (4).

Foi já definida em Portugal a regulamentação da implementação sistemática do rastreio da desnutrição no momento da admissão às unidades prestadoras dos cuidados de saúde, com o Despacho n. ${ }^{\circ} 6634 / 2018$ denominado "Identificação do Risco Nutricional", publicado no Diário da República n. ${ }^{\circ}$ 129/2018, Série II de 2018-0706 e concretizada em Norma Técnica: "Ferramentas a utilizar para a identificação de risco nutricional em Unidades de Saúde Hospitalares dos Serviços Partilhados do Ministério da Saúde" (7-9). O NRS 2002 foi o instrumento recomendado para identificar o risco nutricional nos adultos internados nas Unidades de Saúde Hospitalares.

Existe uma tradução do NRS 2002 para a língua Portuguesa (10), que é aplicada por profissionais de saúde no nosso país. Mas não se encontra publicada a descrição metodológica que foi usada para o seu desenvolvimento. De modo a preservar-se a validade da ferramenta original em traduções, será necessário assegurar a sua equivalência linguística e transcultural. Procedeu-se assim ao desenvolvimento de uma versão para a língua Portuguesa do NRS 2002, em concordância com metodologia recomendada e que fosse linguisticamente e transculturalmente equivalente à original. Este artigo tem como objetivo divulgar este processo e também a versão Portuguesa do NRS 2002.

\section{METODOLOGIA APLICADA}

Numa fase inicial foi solicitada ao titular dos direitos de propriedade intelectual, o Professor Jens Kondrup, a autorização para a tradução e validação para a língua Portuguesa do NRS 2002, tendo esta sido concedida.

Desenvolveu-se uma versão traduzida que apresentasse equivalência transcultural e linguística, recorrendo à metodologia proposta pela Organização Mundial da Saúde (OMS): "Processo de tradução e de adaptação de instrumentos" (11). Este método recorre à tradução avançada e à retrotradução e é composto pelas seguintes etapas: a tradução ( $1 .^{a}$ etapa), a retrotradução efetuada por um ou mais especialistas ( $2 .^{\text {a }}$ etapa), o pré-teste ( $3 .^{\text {a }}$ etapa) e a preparação da versão final (4. ${ }^{a}$ etapa), que se descrevem em seguida (11).

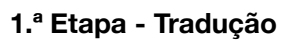

Um dos autores $(L M)$, nutricionista, familiarizado com a terminologia utilizada na ferramenta e com habilidades de entrevista realizou a tradução. De acordo com o preconizado, o tradutor tinha como língua nativa o Português e bons conhecimentos da língua inglesa. Também, e de acordo com a metodologia adotada, o tradutor esforçou-se por ser simples, claro e conciso na formulação das frases.

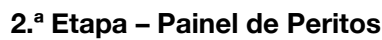

Para se identificar e resolver as expressões/conceitos inadequados da tradução, bem como quaisquer discrepâncias entre a tradução direta e as versões anteriores existentes ou comparáveis das perguntas, caso existissem, convidou-se a participar neste estudo um painel de nutricionistas bilíngues em Português e Inglês (LKS, SCI, FG). Este painel avaliou a primeira tradução e identificou expressões e conceitos inadequados assim como as discrepâncias com o texto original. Os especialistas receberam e pesquisaram materiais que os ajudaram a serem consistentes com as traduções anteriores, questionaram sobre algumas palavras ou expressões e sugeriram alternativas. O resultado desse processo permitiu a produção de uma versão completa traduzida do NRS 2002.

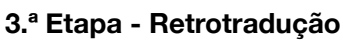

Usando a mesma abordagem descrita no primeiro passo, o NRS 2002 foi então traduzido de volta para o Inglês por um tradutor, cuja língua nativa é o inglês, com formação académica (mestrado) em línguas e sem conhecimento prévio do NRS 2002. Como na tradução inicial descrita na etapa anterior, a ênfase na retrotradução focou-se na equivalência semântica, conceitual e cultural e não na tradução literal. Este tradutor

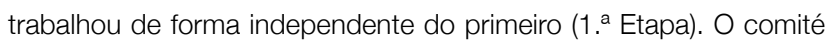
de investigadores discutiu as discrepâncias entre as duas versões e realizou trabalho adicional, até que se produziu uma versão satisfatória - a versão pré-final.

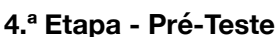

Com o objetivo de os entrevistadores avaliarem se as palavras usadas eram as mais indicadas, efetuou-se o pré-teste (4. ${ }^{\text {a }}$ etapa) desta nova versão do NRS 2002 na população alvo deste sistema, que são os adultos hospitalizados. Convidaram-se nutricionistas com experiência em nutrição clínica superior a dez anos e que desenvolvem a sua atividade em diferentes unidades hospitalares em Portugal continental e insular. Cinco nutricionistas aceitaram participar e aplicaram esta nova versão a cerca de 10 doentes em cada um dos hospitais. Encontram-se descritas na Tabela 1 algumas caraterísticas dos 49 doentes avaliados. Os hospitais em que decorreu este pré-teste encontram-se geograficamente dispersos pelo país e foram avaliados doentes com motivos de internamento do foro médico, cirúrgico e de cuidados intensivos.

Solicitou-se a cada um dos nutricionistas que, se necessário, sugerisse palavras ou expressões alternativas para um item ou expressão. Cada entrevistador forneceu um relatório escrito deste exercício de pré-teste, que incluiu as informações anonimizadas sobre cada NRS 2002 aplicado, o motivo de internamento e as eventuais sugestões de alteração deste instrumento. Não se verificaram omissões no preenchimento do NRS 2002. As sugestões apresentadas foram sobre o fraseado, sobre aspetos gramaticais e também sobre algumas questões de natureza gráfica. Todos foram de opinião de que esta versão Portuguesa do NRS 2002 era compreensível e fácil de aplicar. A documentação e os detalhes sobre cada uma das etapas deste processo estão disponíveis mediante a solicitação ao primeiro autor.

Tabela 1

Informação sobre os participantes no pré-teste

\begin{tabular}{lclc}
\multicolumn{1}{c}{$\begin{array}{c}\text { CENTROS } \\
\text { ENVOLVIDOS }\end{array}$} & DOENTES (N) & $\begin{array}{l}\text { MOTIVO DE } \\
\text { INTERNAMENTO (\%) }\end{array}$ & $\begin{array}{c}\text { RISCO } \\
\text { NUTRICIONAL (\%) }\end{array}$ \\
$\begin{array}{l}\text { Unidade Local de } \\
\text { Saúde do Alto Minho }\end{array}$ & 10 & $\begin{array}{l}\text { Foro médico: 80; } \\
\text { Cancro: 20 }\end{array}$ & 90,0 \\
\hline $\begin{array}{l}\text { Unidade Local de } \\
\text { Saúde da Guarda }\end{array}$ & 9 & $\begin{array}{l}\text { Foro médico: } 77,8 ; \\
\text { Cancro: 22,2 }\end{array}$ & 66,7 \\
\hline $\begin{array}{l}\text { Centro Hospitalar } \\
\text { Universitário do } \\
\text { Algarve }\end{array}$ & 10 & $\begin{array}{l}\text { Foro médico: } 80 ; \\
\text { Cancro: } 20\end{array}$ & 60,0 \\
\hline $\begin{array}{l}\text { Hospital do Divino } \\
\text { Espírito Santo de } \\
\text { Ponta Delgada }\end{array}$ & 10 & $\begin{array}{l}\text { Cuidados intensivos: 80; } \\
\text { Cirurgia: 20 }\end{array}$ & 80,0 \\
\hline $\begin{array}{l}\text { Centro Hospitalar do } \\
\text { Funchal }\end{array}$ & 10 & Foro médico: 100 & 70,0 \\
\hline
\end{tabular}


O comité de investigadores discutiu sobre os relatórios apresentados pelos cinco nutricionistas que colaboraram no pré-teste. Foram analisadas as sugestões decorrentes da interpretação desta versão, que foram incorporadas na versão final do NRS 2002 (Anexo 1). Avaliou-se a relevância cultural desta tradução e também a sua facilidade de compreensão.

Para melhor descrever estes procedimentos, recorreu-se à COSMIN checklist for assessing the methodological quality of studies on measurement properties of health status measurement instruments (12), "Box G: cross cultural validity".

Este trabalho foi alvo de um parecer positivo pela Comissão de Ética do Centro Hospitalar do Algarve (n. $\left.{ }^{\circ} 86 / 19\right)$. Todos os doentes a quem foi aplicada esta versão do NRS 2002, tiveram os resultados comunicados ao profissional de saúde assistente.

A abordagem sistemática adotada para a tradução e adaptação cultural do NRS 2002 recorreu aos princípios e etapas proposta pela OMS (11), apesar de existirem outras propostas metodológicas, nomeadamente o The ITC Guidelines for Translating and Adapting Test (13), o "Report of the ISPOR task force for translation and cultural adaptation (14), que foi em 2017 substituído pelo "Patient-Reported Outcome (PRO) Consortium translation process: consensus development of updated best practices" (15). As razões que estão na base desta nossa opção metodológica prendem-se com a simplicidade e abrangência desta metodologia proposta pela OMS e com o facto de o NRS 2002 se tratar de um sistema simples que deteta a presença de risco de desnutrição, desenvolvido para ser aplicado por profissionais de saúde e não pelo próprio doente.

\section{CONCLUSÕES}

Poderemos assim concluir que este processo resultou numa versão em Português, que é relevante para o rastreio da desnutrição em Portugal (8), e eventualmente em outros países de língua portuguesa.

\section{REFERÊNCIAS BIBLIOGRÁFICAS}

1. Kondrup J, Rasmussen HH, Hamberg O, Stanga Z; Ad Hoc ESPEN Working Group. Nutritional risk screening (NRS 2002): a new method based on an analysis of controlled clinical trials. Clin Nutr. 2003 Jun;22(3):321-36.

2. Rasmussen $\mathrm{HH}$, Holst M, Kondrup J. Measuring nutritional risk in hospitals. Clin Epidemiol. 2010; 21;2:209-16. doi: 10.2147/CLEP.S11265.

3. Hersberger L, Bargetzi L, Bargetzi A, Tribolet P, Fehr R, Baechli V, Geiser M, Deiss M, Gomes F, Kutz A, Kägi-Braun N, Hoess C, Pavlicek V, Schmid S, Bilz S, Sigrist S, Brändle M, Benz C, Henzen C, Nigg M, Thomann R, Brand C, Rutishauser J, Aujesky D, Rodondi N, Donzé J, Stanga Z, Mueller B, Schuetz P. Nutritional risk screening (NRS 2002) is a strong and modifiable predictor risk score for short-term and long-term clinical outcomes: secondary analysis of a prospective randomised trial. Clin Nutr. 2019 Dec 14. doi: 10.1016/j.clnu.2019.11.041.

4. Schuetz P, Fehr R, Baechli V, Geiser M, Deiss M, Gomes F, Kutz A, Tribolet P, Bregenzer T, Braun N, Hoess C, Pavlicek V, Schmid S, Bilz S, Sigrist S, Brändle M, Benz C, Henzen C, Mattmann S, Thomann R, Brand C, Rutishauser J, Aujesky D, Rodondi N, Donzé J, Stanga Z, Mueller B. Individualised nutritional support in medical inpatients at nutritional risk: a randomised clinical trial. Lancet. 2019 Jun 8;393(10188):2312-2321. doi: 10.1016/S0140-6736(18)32776-4.

5. Mendes J, Alves P, Amaral TF. Comparison of nutritional status assessment parameters in predicting length of hospital stay in cancer patients. Clin Nutr. 2014 Jun;33(3):466-70. doi: 10.1016/j.clnu.2013.06.016. Epub 2013 Jul 4.

6. Guerra RS, Fonseca I, Pichel F, Restivo MT, Amaral TF. Usefulness of six diagnostic and screening measures for undernutrition in predicting length of hospital stay: a comparative analysis. J Acad Nutr Diet. 2015 Jun;115(6):927-38. doi: 10.1016/j. jand.2014.11.015.

7. SPMS - Serviços Partilhados do Ministério da Saúde, E.P.E. Norma técnica.
Ferramentas a utilizar para a identificação de risco nutricional em Unidades de Saúde Hospitalares. 31/08/2018. Versão 1.0. Disponível em: http://spms.min-saude.pt/ wp-content/uploads/2015/09/SPMS_2018_NormaTecnica_Avaliac_a_O_Risco_ Nutricional_revisto.pdf.

8. Ministério da Saúde. Direção-Geral da Saúde. Rastreio Nutricional - Documento de apoio à implementação da avaliação do risco nutricional. Lisboa: Direção-Geral da Saúde, 2019. PNPAS e Grupo de Trabalho nomeado pelo Despacho n. 6691/2019, de 26 de julho.

9. Serviços Partilhados do Ministério da Saúde. Ferramentas a utilizar para a identificação de risco nutricional em Unidades de Saúde Hospitalares. 31/08/2018, Versão 1.1, de 26/10/2018: https://spms.min-saude.pt/wp-content/uploads/2018/11/ SPMS_2018_NormaTecnica_Avaliac_a_O_Risco_Nutricional_v1.1.pdf.

10. Luís Matos. Tese de Licenciatura "Desnutrição Hospitalar no Momento da Admissão. Licenciatura em Ciências da Nutrição da Faculdade de Ciências da Nutrição e Alimentação da Universidade do Porto. Porto, 2004.

11. WHO World Health Organization. Process of translation and adaptation of instruments. https://www.who.int/substance_abuse/research_tools/translation/en/. 12. Mokkink LB, Terwee CB, Patrick DL, Alonso J, Stratford PW, Knol DL, Bouter LM, de Vet HC. The COSMIN checklist for assessing the methodological quality of studies on measurement properties of health status measurement instruments: an international Delphi study. Qual Life Res. 2010 May;19(4):539-49. doi: 10.1007/s11136-010-9606-8. 13. International Test Commission. (2017). The ITC Guidelines for Translating and Adapting Tests (Second edition). [www.InTestCom.org].

14. Wild D, Grove A, Martin M, Eremenco S, McElroy S, Verjee-Lorenz A, Erikson $P$ Principles of good practice for the translation and cultural adaptation process for patient-reported outcomes (PRO) measures: report of the ISPOR task force for translation and cultural adaptation. Value Health. 2005. 8(2):94-104. https://doi. org/ 10.1111/j.1524-4733.2005.04054.

15. Eremenco S, Pease S, Mann S, Berry P; PRO Consortium's Process Subcommittee. Patient-Reported Outcome (PRO) Consortium translation process: consensus development of updated best practices. J Patient Rep Outcomes. 2017;2(1):12. doi: 10.1186/s41687-018-0037-6. 


\section{NUTRITIONAL RISK SCREENING (NRS-2002)}

Tabela 1*

Rastreio inicial

\begin{tabular}{ll}
$\mathbf{1}$ & O IMC é $<20,5 ?$ \\
\hline $\mathbf{2}$ & O doente perdeu peso nos últimos 3 meses? \\
\hline $\mathbf{3}$ & O doente teve uma redução na sua ingestão alimentar na última semana? \\
\hline $\mathbf{4}$ & O doente está gravemente doente? (p.e. em terapêutica intensiva) \\
\hline
\end{tabular}

SIM: Se a resposta for "Sim" em qualquer questão, efetuar o rastreio da Tabela 2.

NÃO: Se a resposta for "Não" para todas as questões, o doente é novamente rastreado em intervalos semanais. Se o doente p.e. tem uma cirurgia 'major' programada, é considerado preventivamente um plano de cuidados nutricionais que evite o risco associado.

*Nota do tradutor: de acordo com a publicação original (2003), a Tabela 1 poderá ser aplicada em serviços/unidades de internamento onde previsivelmente a prevalência de risco nutricional seja baixa.

Tabela 2

Rastreio final

\begin{tabular}{|c|c|c|c|c|c|}
\hline \multicolumn{3}{|c|}{ DETERIORAÇÃO DO ESTADO NUTRICIONAL } & \multicolumn{3}{|c|}{ GRAVIDADE DE DOENÇA ( $\approx$ AUMENTO DAS NECESSIDADES) } \\
\hline Ausente & pontuação 0 & Estado nutricional normal & Ausente & pontuação 0 & Necessidades nutricionais normais \\
\hline Ligeira & pontuação 1 & $\begin{array}{l}\text { Perda de peso }>5 \% \text { em } 3 \text { meses } \\
\text { OU Ingestão alimentar abaixo de } \\
50-75 \% \text { das necessidades na } \\
\text { semana anterior }\end{array}$ & Ligeira & pontuação 1 & $\begin{array}{l}\text { Fratura da anca*, Doentes } \\
\text { crónicos, em particular com } \\
\text { complicações agudas: cirrose }^{\star} \text {, } \\
\text { DPOC }{ }^{\star}, \text { Hemodiálise crónica, } \\
\text { diabetes, oncologia. }\end{array}$ \\
\hline Moderada & pontuação 2 & $\begin{array}{l}\text { Perda de peso > 5\% em } 2 \\
\text { meses OU IMC } 18,5-20,5+ \\
\text { deterioração do estado geral OU } \\
\text { Ingestão alimentar } 25-60 \% \text { das } \\
\text { necessidades na semana anterior }\end{array}$ & Moderada & pontuação 2 & $\begin{array}{l}\text { Cirurgia abdominal 'major”, } \mathrm{AVC}^{*} \text {, } \\
\text { Pneumonia grave, malignidade } \\
\text { hematológica }\end{array}$ \\
\hline Grave & pontuação 3 & $\begin{array}{l}\text { Perda de peso > } 5 \% \text { em } 1 \text { mês } \\
\text { (>15\% em } 3 \text { meses) OU IMC < } 18,5 \\
\text { + deterioração do estado geral } \\
\text { OU Ingestão alimentar } 0-25 \% \text { das } \\
\text { necessidades na semana anterior }\end{array}$ & Grave & pontuação 3 & $\begin{array}{l}\text { Lesão craneoencefálica*, } \\
\text { Transplante de medula óssea } \\
\text { Doentes de cuidados intensivos } \\
(\text { APACHE > 10) }\end{array}$ \\
\hline Pontuação & & + & Pontuação & & = Pontuação total: \\
\hline
\end{tabular}

Pontuação $\geq 3$ : o doente está em risco nutricional e é iniciado um plano de cuidados nutricionais

Pontuação < 3: repetir rastreio semanalmente. Se o doente p.e. tem uma cirurgia 'major' programada, é considerado preventivamente um plano de cuidados nutricionais que evite o risco associado.

* Indica que um ensaio clínico suporta especificamente a inclusão da patologia nessa categoria de gravidade.

Os diagnósticos apresentados em itálico são baseados nos padrões de gravidade descritos abaixo.

O NRS-2002 é baseado na interpretação de ensaios clínicos randomizados disponíveis. (Nota do tradutor: até à data de publicação do original, 2003)

Risco Nutricional é definido pelo estado nutricional atual e pelo risco de deterioração do estado atual, devido a um aumento das necessidades nutricionais causado por stress metabólico associado à condição clínica.

\begin{tabular}{cl} 
ESTÁ INDICADO UM PLANO DE CUIDADOS NUTRICIONAIS PARA TODOS OS DOENTES QUE ESTÃO COM: \\
$\mathbf{1} \quad$ Desnutrição grave (pontuação $=3$ ) \\
\hline $\mathbf{2}$ & Doença grave (pontuação $=3$ ) \\
\hline $\mathbf{3}$ & Desnutrição moderada (pontuação $=$ 2) e Doença ligeira (pontuação $=1$ ) \\
\hline $\mathbf{4}$ & Desnutrição ligeira (pontuação $=1$ ) e Doença moderada (pontuação $=2)$ \\
\hline
\end{tabular}

\section{Padrões de gravidade de doença:}

Pontuação = 1: doente com doença crónica, admitido no hospital por complicações. O doente está fragilizado, mas faz "levante do leito" regularmente. As necessidades proteicas estão aumentadas, mas podem ser atingidas através de alimentação ou suplementação orais, na maioria dos casos.

Pontuação = 2: doente acamado devido a doença, p.e. após cirurgia abdominal 'major'. As necessidades proteicas estão substancialmente aumentadas, mas podem ser atingidas, embora em muitos casos seja necessária nutrição artificial.

Pontuação = 3: doente internado em cuidados intensivos, com necessidade de ventilação assistida, etc. As necessidades proteicas estão aumentadas e não podem ser atingidas, mesmo com nutrição artificial. O catabolismo proteico e perda de azoto podem ser significativamente atenuados. 